\title{
Steric effect: A quantitative description from density functional theory
}

\author{
Shubin Liu ${ }^{a)}$ \\ The Renaissance Computing Institute (RENCI), University of North Carolina, Chapel Hill, \\ North Corolina 27599-3420 and Division of Research Computing, Information Technology Services, \\ University of North Carolina, Chapel Hill, North Carolina 27599-3420
}

(Received 19 April 2007; accepted 14 May 2007; published online 22 June 2007)

\begin{abstract}
The concepts of steric energy, steric potential, and steric charge are introduced within the density functional theory framework. The steric energy, representing a hypothetical state with all electrons packed into the lowest orbital and other effects entirely excluded, is a measure of the intrinsic space occupied by an electronic system. It is exclusive, repulsive, and extensive, and it vanishes for homogeneous electron gas. When Bader's zero-flux boundary condition is adopted, atoms in molecules are found to achieve balanced steric repulsion among one another with vanished steric energy density interfaces. A few molecular systems involving conformation changes and chemical reactions have been investigated to examine the relative contribution of the steric and other effects, providing insights for a few controversial topics from a different perspective. () 2007 American Institute of Physics. [DOI: 10.1063/1.2747247]
\end{abstract}

Steric effect is one of the most widely used concepts in chemistry. ${ }^{1}$ It originates from the fact that each atom in a molecule occupies a certain amount of space. When atoms are brought together, hindrance will be induced in the expense of shape, energy, reactivity, etc. While qualitative reference to the steric effect is commonplace in the literature, a satisfactory quantification is still lacking. Here, we propose a quantitative description for the effect from the framework of density functional theory (DFT). ${ }^{2,3}$ Its appealing properties are illustrated and relevant to Bader's atoms in molecules ${ }^{4}$ (AIM) approach revealed. A few molecular systems involving conformation change, chemical reaction, and the anomeric effect will be investigated to examine the relative contribution of the steric and other effects, providing insights for a few controversial topics from a different perspective.

An admissible definition of the steric effect needs to show that the effect is repulsive in nature and extensive in size. It must be repulsive because it relates to the occupied space, and overlap with others will result in objection. It must be extensive in order to conform to the notion that the bulkier the system, the larger the steric effect. Meanwhile, although the steric effect pertains to the space undertaken by an atom or molecule, others such as quantum and electrostatic effects also contribute to the constitution of a molecular framework. The contribution from the quantum effect ${ }^{5,6}$ comes from the Pauli exclusion principle (Fermi hole $)^{7}$ and dynamic electron correlation effect (Coulomb hole), ${ }^{8,9}$ preventing both same-spin and opposite-spin electrons from coming together. Electrostatic interactions such as classical electron-electron and nuclear-nuclear Coulomb repulsions keep both electrons and nuclei in a molecule in check and thus also contribute to the composition of the molecular scaffold. To be exclusive, a pertinent depiction of the steric effect should be able to set aside the shares from other effects.

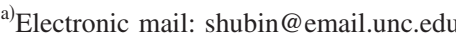

Now, we start with the exclusiveness hypothesis by assuming that the total energy density functional of an atom and molecule can alternatively be partitioned as

$$
E[\rho] \equiv E_{s}[\rho]+E_{e}[\rho]+E_{q}[\rho],
$$

where $E_{s}[\rho], E_{e}[\rho]$, and $E_{q}[\rho]$ stand for the independent energy contribution from the steric, electrostatic, and quantum effects, respectively. In DFT, ${ }^{2,3}$ we know that

$$
E[\rho]=T_{S}[\rho]+V_{\mathrm{ne}}[\rho]+J[\rho]+V_{\mathrm{nn}}[\rho]+E_{\mathrm{xc}}[\rho],
$$

where $T_{S}[\rho], V_{\text {ne }}[\rho], J[\rho], V_{\text {nn }}[\rho]$, and $E_{\text {xc }}[\rho]$ represent the noninteracting kinetic, nuclear-electron attraction, classical interelectron Coulomb repulsion, nuclear-nuclear repulsion, and exchange-correlation energy density functionals, respectively. Three terms in Eq. (2), $V_{\text {ne }}[\rho], J[\rho]$, and $V_{\text {nn }}[\rho]$, are of the electrostatic nature. Hence,

$$
E_{e}[\rho]=V_{\mathrm{ne}}[\rho]+J[\rho]+V_{\mathrm{nn}}[\rho] .
$$

For the quantum contribution, there are two sources. One is from $E_{\mathrm{xc}}[\rho]$ and the other from the noninteracting kinetic energy $T_{S}[\rho],{ }^{10-14} E_{\text {Pauli }}[\rho] \equiv T_{S}[\rho]-T_{W}[\rho]$, called the Pauli energy, where $T_{W}[\rho]$ is the Weizsäcker kinetic energy, ${ }^{2,3,15}$

$$
T_{W}[\rho]=\frac{1}{8} \int \frac{|\nabla \rho(\mathbf{r})|^{2}}{\rho(\mathbf{r})} d \mathbf{r} .
$$

The Pauli energy $E_{\text {Pauli }}[\rho]$ represents the portion of the kinetic energy that embodies all the effects from the antisymmetric requirement of the total wave function by the Pauli exclusion principle ${ }^{10-14}$. Notice that there is also a kinetic counterpart of the dynamic correlation effect, $T_{c}[\rho]$, already included in $\left.E_{\mathrm{xc}}[\rho]\right]^{2,3,16-18}$ Put together,

$$
E_{q}[\rho]=E_{\text {xс }}[\rho]+E_{\text {Pauli }}[\rho]=E_{\text {xс }}[\rho]+T_{S}[\rho]-T_{W}[\rho] .
$$

With Eqs. (1)-(3) and (5), there comes our definition of the steric energy,

$$
E_{s}[\rho] \equiv E[\rho]-E_{e}[\rho]-E_{q}[\rho]=T_{W}[\rho] .
$$




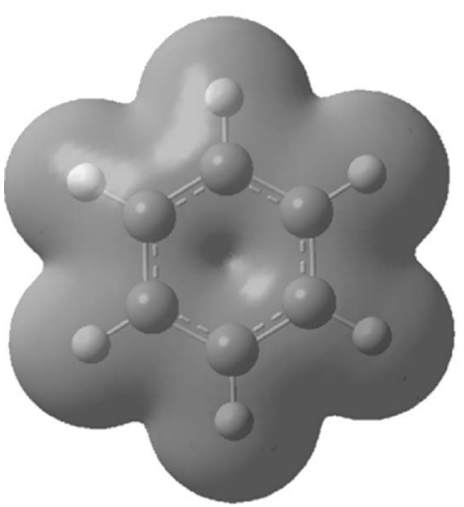

(a)

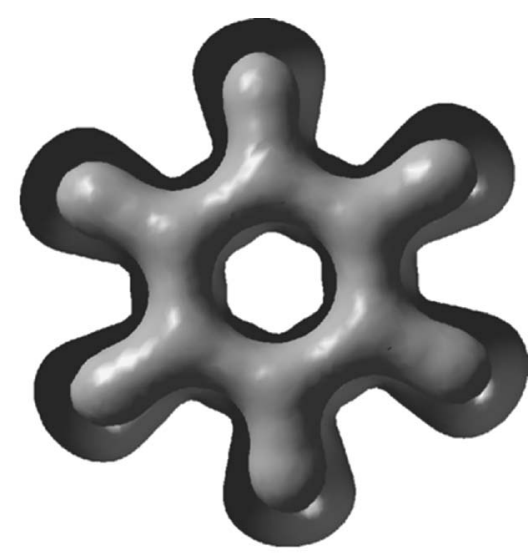

(b)
Physically speaking, if electrons in an atom or molecule were bosons, they would be squeezed into the lowest orbital in the ground state. Assuming that the electron density of the hypothetical state is the same as the Fermion state $\rho(\mathbf{r})$, the total wave function of the state will simply be $\sqrt{ }[\rho(\mathbf{r}) / N](N$ being the number of electrons). Excluding electrostatic and quantum contributions, the total energy of the conjectural system is then $T_{W}[\rho]$. The space withheld by the state and represented by $E_{s}[\rho]$ is hence an intrinsic property of the system. In this regard, $E_{s}[\rho]$ is a measure of the intrinsic dimensions upheld by the system with the contributions from other effects, quantum and electrostatic, completely excluded. In addition, the definition is consistent with the original Weisskopf's attribution ${ }^{5}$ of the steric effect to the "kinetic energy pressure" because $T_{W}[\rho]$ is one kinetic energy.

The following properties of the above definition are in order. The steric energy $E_{s}[\rho]$ is a universal density functional, always a lower bound to the true kinetic energy ${ }^{19-21}$ and always nonnegative. Given the nature of the atomic and molecular electron density, ${ }^{22-24}$ the steric energy density, $1 / 8|\nabla \rho(\mathbf{r})|^{2} / \rho(\mathbf{r})$, is nowhere negative in space. Secondly, $E_{s}[\rho]$ is extensive. That is, the larger the system, the larger the steric energy. This is because the steric energy density functional is homogeneous of degree 1 in density scaling, ${ }^{2,16,25}$ satisfying

$$
\int \rho(\mathbf{r}) \frac{\delta E_{S}[\rho]}{\delta \rho(\mathbf{r})} d \mathbf{r}=E_{s}[\rho]
$$

In other words, $E_{s}[\gamma \rho]=\gamma E_{s}[\rho]$ for $0 \leqslant \gamma \leqslant 1$. For homogeneous electron gas, ${ }^{2,3}$ where the density is everywhere uniform with $\nabla \rho(\mathbf{r})=0$, the steric energy is zero, indicating that there (correctly) exists no steric repulsion in homogeneous electron gas. For one-electron cases ${ }^{23}$ such as $\mathrm{H}$ and $\mathrm{H}_{2}^{+}$, one has $E_{s}=-E$, showing that the value of the steric and total energies is the same but the two quantities have opposite signs. More importantly, if Bader's definition of the zero-flux boundary condition ${ }^{4}$ is adopted, the concept of atoms in a molecule can then be established with the characteristic that the atoms are interfaced with each other with the surface of a vanished steric energy density, exhibiting that atoms in a molecule acquire balanced steric repulsion among one another.
We introduce the steric potential $v_{s}(\mathbf{r})$ as the functional derivative of the steric energy $E_{s}[\rho]$ with respect to the total electron density,

$$
v_{s}(\mathbf{r})=\frac{\delta E_{s}[\rho]}{\delta \rho(\mathbf{r})}=\frac{1}{8} \frac{|\nabla \rho(\mathbf{r})|^{2}}{\rho^{2}(\mathbf{r})}-\frac{1}{4} \frac{\nabla^{2} \rho(\mathbf{r})}{\rho(\mathbf{r})}
$$

and the steric charge $q_{s}(\mathbf{r})$, obeying ${ }^{26}$

$$
\nabla^{2} v_{s}(\mathbf{r})=-4 \pi q_{s}(\mathbf{r}) .
$$

It can readily be shown that $v_{s}(\mathbf{r})$ is no longer positive everywhere. Though singular at the nuclear cusp, ${ }^{27}$ it approaches to the finite number $-I$, ionization potential, asymptotically. ${ }^{28}$ Also, the total steric charge is zero, $\int q_{s}(\mathbf{r}) d \mathbf{r}=0$. At the zero-flux boundary, ${ }^{4}$ where the steric energy density vanishes, the steric potential in Eq. (8) is dictated by the only term of the density Laplacian, $\nabla^{2} \rho(\mathbf{r})$, suggesting that this quantity can play vastly important roles in chemical processes as have already been showcased by Bader et al..$^{4,29}$

Figure 1 as illustrative example shows contour plots of the steric energy density and steric potential for the benzene molecule. The steric energy density is shown to be nonnegative and preserves the shape of the molecule, whereas for the steric potential it is repulsive (gray colored) near the nuclear and bonding regions and becomes attractive (dark colored) outside. The shape of both the repulsive and attractive domains resembles that of the molecule that they encompass.

A prompt application of the present description is to analyze the relative importance of the three energy components in Eq. (1) for the process of isomer conformation changes and chemical reactions. ${ }^{30}$ There continues to be a lot of controversy in the literature. ${ }^{31-33}$ Our present work provides insights from a rather different perspective. Table I shows some of the results for a few molecular systems.

Our first example is the protonated methane ${ }^{34} \mathrm{CH}_{5}^{+}$, which is known to have a few isomers. The two most stable conformations are $C_{s}(\mathrm{I})$ and $C_{s}(\mathrm{II})$. From Table I, we find that the energy difference between the two is dominated by the steric hindrance. Our next example is about the two conformers, eclipsed and staggered, of ethane, ${ }^{31-33}$ where we notice that the energy difference between them comes from the positive contribution of electrostatic and steric interactions, with the latter larger in magnitude, indicating that the 
TABLE I. The total energy difference and its partition according to Eq. (1) of the text for a number of chemical processes including isomer conformation changes, bonding formation, chemical reactions and the anomeric effect. Units in $\mathrm{kcal} / \mathrm{mol}$.

\begin{tabular}{|c|c|c|c|c|}
\hline & \multicolumn{3}{|c|}{ Energy components } & \multirow{2}{*}{$\begin{array}{c}\text { Total energy } \\
\text { difference }\end{array}$} \\
\hline & Electrostatic & Quantum & Steric & \\
\hline $\begin{array}{l}\mathrm{CH}_{5}^{+} \\
C_{S}(\mathrm{II})-C_{S}(\mathrm{I})\end{array}$ & -0.55 & -0.74 & 1.67 & 0.38 \\
\hline $\begin{array}{l}\mathrm{CH}_{3}-\mathrm{CH}_{3} \\
\text { eclipsed-staggered }\end{array}$ & 5.95 & -11.41 & 7.71 & 2.25 \\
\hline $\begin{array}{l}\mathrm{CH}_{3}-\mathrm{CH}_{2}-\mathrm{CH}_{2}-\mathrm{CH}_{3} \\
\text { eclipsed-staggered }^{\mathrm{a}}\end{array}$ & -28.76 & 82.32 & -43.28 & 10.27 \\
\hline $\begin{array}{l}\mathrm{H}-\mathrm{H} \text { bond } \\
2.0-1.0 \AA\end{array}$ & 123.81 & 71.68 & -106.76 & 88.72 \\
\hline $\begin{array}{l}\mathrm{F}-\mathrm{F} \\
2.0-1.0 \AA\end{array}$ & -987.81 & 2402.04 & -1241.02 & 173.21 \\
\hline $\begin{array}{l}\mathrm{Na}-\mathrm{Cl} \text { bond } \\
5.0-2.0 \AA\end{array}$ & 268.31 & -449.94 & 214.27 & 32.64 \\
\hline $\begin{array}{l}\mathrm{He}-\mathrm{He} \\
3.0-2.0 \AA\end{array}$ & 126.31 & -285.37 & 70.46 & -88.59 \\
\hline $\begin{array}{l}\mathrm{Ne}-\mathrm{Ne} \\
3.0-2.0 \AA\end{array}$ & 50.46 & -161.86 & 102.38 & -9.02 \\
\hline $\begin{array}{l}\mathrm{BH}_{3}-\mathrm{CO} \text { bond } \\
5.0-1.5 \AA\end{array}$ & 35.55 & -622.53 & 619.13 & 32.15 \\
\hline $\begin{array}{l}\mathrm{H}_{2} \mathrm{O}-\mathrm{Li}^{+} \text {bond } \\
5.5-2.0 \AA\end{array}$ & 8.32 & -4132.61 & 4154.27 & 29.98 \\
\hline $\begin{array}{l}\mathrm{Cl}-\mathrm{CH}_{3}-\mathrm{Cl}^{-} \\
\text {TS-reactant }\end{array}$ & 4.50 & -89.97 & 88.96 & 4.78 \\
\hline $\begin{array}{l}\mathrm{HCHO} \Rightarrow \mathrm{H} 2+\mathrm{CO} \\
\text { TS-reactant }\end{array}$ & 91.93 & -5.29 & -9.04 & 77.59 \\
\hline $\begin{array}{l}\mathrm{HCN} \Rightarrow \mathrm{HNC} \\
\text { TS-reactant }\end{array}$ & 66.50 & -97.34 & 76.07 & 45.23 \\
\hline $\begin{array}{l}\mathrm{HNC} \Rightarrow \mathrm{HCN} \\
\text { TS-reactant }\end{array}$ & 27.40 & -105.38 & 107.62 & 29.64 \\
\hline $\begin{array}{l}\mathrm{C}_{6} \mathrm{H}_{11}-\mathrm{F} \\
\text { Axial-equatorial }\end{array}$ & -4.73 & 22.50 & -15.63 & 2.14 \\
\hline $\begin{array}{l}\mathrm{C}_{5} \mathrm{H}_{9} \mathrm{O}-\mathrm{F} \\
\text { Axial-equatorial }\end{array}$ & -7.39 & 1.21 & 4.57 & -1.61 \\
\hline
\end{tabular}

${ }^{\mathrm{a}}$ Reference 30 .

eclipsed conformer possesses larger steric repulsion. The negative contribution comes solely from the quantum effect. For the same two conformers of $n$-butane, however, the situation is different, with electrostatic and steric effects contributing negatively and the dominant factor from the quantum effect. $^{35}$

Other systems that we have looked into include different categories of chemical bonds, transition states, and the anomeric effect. In $\mathrm{H}_{2}$, as shown in the table, when two hydrogen atoms get closer, more steric repulsion is induced, but it is compensated by stronger quantum and electrostatic interactions. The same is true in the F-F bond formation for $\mathrm{F}_{2}$ dimer, but as the two $\mathrm{F}$ atoms get too close, the electrostatic interaction becomes repulsive and the dominant contribution comes exclusively from the quantum effect. For the ionic bond formation, taking $\mathrm{Na}-\mathrm{Cl}$ as an example in Table I, steric and electrostatic effects are positively contributed to compensate the negatively contributed quantum effect. We next consider the homogeneous dimer of two noble atoms, $\mathrm{He}-\mathrm{He}$ and $\mathrm{Ne}-\mathrm{Ne}$. One finds that the quantum effect dominates the negative interaction energy as the two atoms get closer, consistent with the findings that van der Waals interactions are of quantum nature. ${ }^{36-39}$ For the breakdown of $\mathrm{BH}_{3}-\mathrm{CO}$ and $\mathrm{H}_{2} \mathrm{O}-\mathrm{Li}^{+}$complexes, we found that electrostatic interactions play a minor role, the dominant term is the positive contribution from the steric effect and the quantum effect is a hindrance.

When a chemical reaction takes place, the three components in Eq. (1) contribute differently to the barrier height of the transition state. Table I also displays the analysis for three reactions, $S_{N} 2$ nucleophilic self-exchange reaction of $\mathrm{Cl}^{-}$ $+\mathrm{CH}_{3} \mathrm{Cl}$, $\mathrm{HCHO}$ conversion to $\mathrm{H}_{2}+\mathrm{CO}$, and $\mathrm{HCN} \Leftrightarrow \mathrm{HNC}$ isomerization. We found that different components become dominant in different reactions. For example, electrostatic interactions control the barrier height of the $\mathrm{HCHO}$ conversion reaction, whereas in the $S_{N} 2$ reaction the steric and quantum effects are the controlling factors.

Our last example in Table I is concerned with the anomeric effect. ${ }^{40}$ We first consider fluorine substituted cyclohexane. The energy difference between the two conformers where $\mathrm{F}$ is in axial and equatorial positions, respectively, is mainly governed by the quantum and steric effects, confirming that it is really a stereoelectronic effect. However, the steric contribution is negative and quantum contribution is positive, indicating that there is less steric hindrance in the axial position. When a neighboring carbon atom is replaced by oxygen, the anomeric effect will come into play. ${ }^{40}$ As shown in the last row of the table, the dominant factors are changed to the electrostatic and steric interactions, with the positive contribution from steric repulsion and negative one from the electrostatic interaction. In our view, this effect is really of stereoelectrostatic nature with little contribution from the quantum effect. The conformer with the fluorine atom in the axial position in this case is found to have larger steric repulsion.

We anticipate that the concepts proposed in this work can readily be applied to other systems, especially those with regioselective, diastereoselective, and enantioselective characteristics and ones with stereoelectronic effects in play. With Bader's zero-flux boundary condition, one can unambiguously characterize the atomic contribution of the energy components in Eq. (1). It is also possible to decompose their corresponding potential and charge into atomic contributions.

A few theoretical developments are also possible. First, with the quantum energy in Eq. (5), one can similarly define the quantum potential $v_{q}(\mathbf{r})$ and quantum charge $q_{q}(\mathbf{r})$ with the help that ${ }^{2,3,41}$

$$
\frac{\delta T_{S}[\rho]}{\delta \rho(\mathbf{r})}=\mu-v_{\mathrm{eff}}^{\mathrm{KS}}(\mathbf{r}),
$$

where $\mu$ is chemical potential and $v_{\text {eff }}^{\mathrm{KS}}(\mathbf{r})$ is the effective Kohn-Sham potential ${ }^{2,3}$

$$
v_{\mathrm{eff}}^{\mathrm{KS}}(\mathbf{r})=v_{\mathrm{ext}}(\mathbf{r})+v_{J}(\mathbf{r})+v_{\mathrm{xc}}(\mathbf{r})
$$

where $v_{\text {ext }}(\mathbf{r}), v_{J}(\mathbf{r})$, and $v_{\text {xc }}(\mathbf{r})$ denote the functional derivative of $V_{\text {ne }}[\rho], J[\rho]$, and $E_{\mathrm{xc}}[\rho]$ in Eq. (2), respectively. With Eqs. (5), (10), and (11), one has 


$$
v_{q}(\mathbf{r})=\frac{\delta E_{q}[\rho]}{\delta \rho(\mathbf{r})}=\mu-v_{\mathrm{ext}}(\mathbf{r})-v_{J}(\mathbf{r})-v_{s}(\mathbf{r})
$$

and

$$
\nabla^{2} v_{q}(\mathbf{r})=-4 \pi q_{q}(\mathbf{r}) .
$$

Notice that in Eq. (12), all terms at the right-hand side are explicitly known and no exchange-correlation potential is involved. The Euler equation for Eq. (1) in the orbital-less DFT approach ${ }^{11-13,16,41,42}$ then becomes

$$
\left\{-\frac{1}{2} \nabla^{2}+v_{e}(\mathbf{r})+v_{q}(\mathbf{r})\right\} \rho^{1 / 2}(\mathbf{r})=\mu \rho^{1 / 2}(\mathbf{r}),
$$

where the electrostatic potential $v_{e}(\mathbf{r})=v_{\text {ext }}(\mathbf{r})+v_{J}(\mathbf{r})$. There is only one unknown, $v_{\mathrm{q}}(\mathbf{r})$, to approximate and no knowledge of $E_{\mathrm{xc}}[\rho]$ and $v_{\mathrm{xc}}(\mathbf{r})$ is required in Eq. (12). Equation (14) thus provides an alternative approach for the orbital-less DFT.

Another aspect of the development is to apply Eq. (1) to conceptual DFT to investigate energy component contributions to the reactivity indices. For example, for the chemical potential $\mu$, with Eq. (1), one has

$$
\begin{aligned}
\mu=\left(\frac{\partial E}{\partial N}\right)_{v}= & \int v_{s}(\mathbf{r}) f(\mathbf{r}) d \mathbf{r} \\
& +\int v_{e}(\mathbf{r}) f(\mathbf{r}) d \mathbf{r}+\int v_{q}(\mathbf{r}) f(\mathbf{r}) d \mathbf{r},
\end{aligned}
$$

where $v_{x}(x=s, e, q)$ are the three potentials from Eqs. (3), (5), and (6), respectively, and $\mathrm{f}(\mathbf{r})$ is the Fukui function ${ }^{42}$

$$
f(\mathbf{r})=\left(\frac{\partial \rho(\mathbf{r})}{\partial N}\right)_{v}
$$

It is worthwhile to investigate the relative importance of the three terms in Eq. (15) in chemical processes. The same idea applies to other DFT indices. ${ }^{43}$ One can also perform the same analysis at the atomic level of resolution with the help of Bader's zero-flux boundary condition.

Although we restrict ourselves only to systems in the ground state, extension of the present work to condensed phases, excited states, multiplets, and time-dependent systems is straightforward.

In summary, a quantitative description of the steric effect in terms of steric energy, steric potential, and steric charge has been proposed in the present work within the DFT framework. We introduce the Weizsäcker kinetic energy as the measure of the intrinsic space occupied by an atom or molecule. It is the total energy of a postulated state where all electrons are squeezed into the lowest orbital and all other effects are completely omitted. It has been shown that besides being exclusive, the steric energy is repulsive and extensive, and it vanishes for the homogeneous electron gas. When Bader's zero-flux boundary condition is adopted, the concept of atoms in molecules will be established with the feature that the atoms maintain balanced steric repulsion with one another by sharing the surface of a vanished steric energy density. We have applied the idea to a number of systems involving conformation changes, chemical reactions, and the anomeric effect. Our results provide insights from a different perspective on a number of controversial topics. Other possible applications and developments have also been outlined.

We conclude by noting in passing that there is no physically observable variable associated with the steric effect and thus, quantum mechanically speaking, it is a noumenon, ${ }^{44-49}$ an object, though chemically significant and conceptually relevant in understanding the behavior of molecules, of purely rational apprehension and intellectual intuition. Indeed, any energy decomposition scheme that employs an atomic picture has the difficulty in defining local energies or, more generally, local properties of an operator. ${ }^{50-55}$ For this reason, other quantitative descriptions of the effect, such as the one ${ }^{6,32}$ employing the Pauli exclusion principle as the characterization, are quite likely. In our current approach, we assumed the validity of the exclusiveness hypothesis of the total energy partition, Eq. (1). All contributions from the Pauli exclusion principle are categorically included into the quantum effect. Besides this fundamental difference between the two descriptions, attractive properties from our present approach, such as repulsiveness and extensiveness as well as its link to Bader's AIM method, have been revealed. In addition, we provided an explicit and self-consistent definition for the energy, potential, and even charge associated with the steric effect, which can afford a wealth of in-depth information about different chemical processes. Despite these rudimentary differences, a systematic comparison between the two descriptions is of interest, whose results will be reported elsewhere. database, http://isiknowledge.com, when the keyword of "steric" was searched. With the keyword of "stereo"," more than 81000 hits have been come across in the title search.

${ }^{2}$ R. G. Parr and W. Yang, Density Functional Theory of Atoms and Molecules (Oxford University Press, Oxford, 1989).

${ }^{3}$ R. M. Dreizler and E. K. U. Gross, Density Functional Theory: An Approach to the Quantum Many-Body Problem (Springer-Verlag, New York, 1990).

${ }^{4}$ R. F. W. Bader, Atoms in Molecules: A Quantum Theory (Oxford University Press, Oxford, 1990).

${ }^{5}$ V. F. Weisskopf, Science 187, 605 (1975).

${ }^{6}$ J. K. Badenhoop and F. Weinhold, J. Chem. Phys. 107, 5406 (1997).

${ }^{7}$ W. L. Luken and D. N. Beratan, Theor. Chem. Acc. 61, 1432 (1982).

${ }^{8}$ R. Benesch and V. H. Smith, J. Chem. Phys. 55, 482 (1971).

${ }^{9}$ R. J. Boyd and M. C. Yee, J. Chem. Phys. 77, 3578 (1982).

${ }^{10}$ N. H. March, Phys. Lett. 113A, 476 (1986).

${ }^{11}$ M. Levy and H. Ou-Yang, Phys. Rev. A 38, 625 (1988).

${ }^{12}$ C. Herring and M. Chopra, Phys. Rev. A 37, 31 (1988).

${ }^{13}$ A. Savin, A. D. Becke, J. Flad, R. Nesper, H. Preuss, and H. G. von Schnering, Angew. Chem., Int. Ed. 30, 409 (1991).

${ }^{14}$ A. Holas and N. H. March, Phys. Rev. A 44, 5521 (1991).

${ }^{15}$ C. F. von Weizsäcker, Z. Phys. 96, 431 (1935).

${ }^{16}$ M. Levy and J. P. Perdew, Phys. Rev. A 32, 2010 (1985).

${ }^{17}$ S. B. Liu and R. G. Parr, Phys. Rev. A 53, 2211 (1996).

${ }^{18}$ S. B. Liu, R. C. Morrison, and R. G. Parr, J. Chem. Phys. 125, 174109 (2006).

${ }^{19}$ M. Hoffmann-Ostenhof and T. Hoffmann-Ostenhof, Phys. Rev. A 16, 1782 (1977)

${ }^{20}$ S. B. Sears, R. G. Parr, and U. Dinur, Isr. J. Chem. 19, 165 (1980).

${ }^{21}$ M. Levy, J. P. Perdew, and V. Sahni, Phys. Rev. A 30, 2745 (1984).

${ }^{22}$ W. P. Wang and R. G. Parr, Phys. Rev. A 16, 891 (1977).

${ }^{23}$ S. B. Liu and R. G. Parr, J. Comput. Chem. 20, 2 (1999).

${ }^{24}$ S. B. Liu, Int. J. Quantum Chem. 106, 1762 (2006).

${ }^{25}$ S. B. Liu and R. G. Parr, Phys. Rev. A 55, 1792 (1997).

${ }^{26}$ S. B. Liu, P. W. Ayers, and R. G. Parr, J. Chem. Phys. 111, 6197 (1999).

${ }^{27}$ Z. Janosfalvi, K. D. Sen, and A. Nagy, Phys. Lett. A 344, 1 (2005). 
${ }^{28}$ Z. Z. Zhang, S. B. Liu, and Y. A. Wang, Chem. Phys. Lett. 258, 30 (1996).

${ }^{29}$ R. F. W. Bader, R. J. Gillespie, and P. J. MacDougall, J. Am. Chem. Soc. 110, 7329 (1988).

${ }^{30}$ All calculations were performed using the BLYP functional and doublezeta plus polarization quality numerical basis set. For more information, see W. Yang, J. Chem. Phys. 94, 1208 (1991); S. B. Liu, J. M. PerezJorda, and W. Yang, ibid. 112, 1634 (2000).

${ }^{31}$ V. Pophristic and L. Goodman, Nature (London) 411, 565 (2001).

${ }^{32}$ F. M. Bickelhaupt and E. J. Baerends, Angew. Chem., Int. Ed. 42, 4183 (2003).

${ }^{33}$ F. Weinhold,. Angew. Chem., Int. Ed. 42, 4188 (2003).

${ }^{34}$ E. T. White, J. Tang, and T. Oka, Science 284, 135 (1999).

${ }^{35}$ Using the definition from Ref. 7, one also obtains the same (negative) sign of the steric contribution for the two n-butane isomers. With the B3LYP/6-311+G(d) geometry, the total steric energy calculated by NBO 5.0 (based on Ref. 7) for the staggered conformation is $127.09 \mathrm{kcal} / \mathrm{mol}$ and that of the eclipsed conformer is $119.73 \mathrm{kcal} / \mathrm{mol}$, giving $-7.36 \mathrm{kcal} / \mathrm{mol}$ of the steric energy difference (eclipsed-staggered) between the two.

${ }^{36}$ A. D. Becke and E. R. Johnson, J. Chem. Phys. 122, 154104 (2005).

${ }^{37}$ E. R. Johnson and A. D. Becke, J. Chem. Phys. 123, 024101 (2005).
${ }^{38}$ A. D. Becke and E. R. Johnson, J. Chem. Phys. 124, 014104 (2005).

${ }^{39}$ E. R. Johnson and A. D. Becke, J. Chem. Phys. 124, 174104 (2006).

${ }^{40}$ E. Juaristi and G. Cuevas, The Anomeric Effect (CRC, Boca Raton, FL, 1995).

${ }^{41}$ S. B. Liu and P. W. Ayers, Phys. Rev. A 70, 022501 (2004).

${ }^{42}$ P. W. Ayers and S. B. Liu, Phys. Rev. A 75, 022514 (2007).

${ }^{43}$ P. Geerlings, F. De Proft, and W. Langenaeker, Chem. Rev. (Washington, D.C.) 103, 1793 (2003).

${ }^{44}$ R. G. Parr, P. W. Ayers, and Roman F. Nalewajski, J. Phys. Chem. A 109, 3957 (2005).

${ }^{45}$ S. Shahbazian and M. Zahedi, Found. Chem. 8, 37 (2006).

${ }^{46} \mathrm{G}$. Frenking and A. Krapp, J. Comput. Chem. 28, 15 (2007).

${ }^{47}$ R. Hoffmann, J. Mol. Struct.: THEOCHEM 424, 1 (1998).

${ }^{48}$ P. W. Ayers, J. Chem. Phys. 113, 10886 (2000).

${ }^{49}$ P. W. Ayers, Faraday Discuss. 135, 161 (2007).

${ }^{50}$ L. Cohen, Phys. Lett. A 212, 315 (1996).

${ }^{51}$ L. Cohen, J. Chem. Phys. 80, 4277 (1984).

${ }^{52}$ L. Cohen and Y. I. Zaparovanny, J. Math. Phys. 21, 794 (1980).

${ }^{53}$ L. Cohen, J. Chem. Phys. 70, 788 (1979).

${ }^{54}$ L. Cohen, J. Math. Phys. 7, 781 (1966).

${ }^{55}$ P. W. Ayers, R. G. Parr, and A. Nagy, Int. J. Quantum Chem. 90, 309 (2002). 